\title{
Numerical simulation of acoustic wake effect in acoustic agglomeration under Oseen flow condition
}

\author{
ZHANG GuangXue ${ }^{1 *}$, LIU JianZhong ${ }^{2}$, WANG Jie ${ }^{2}$, ZHOU JunHu ${ }^{2} \&$ CEN $\mathrm{KeFa}^{2}$ \\ ${ }^{1}$ Institute for Energy Engineering, China Jiliang University, Hangzhou 310018, China; \\ ${ }^{2}$ Institute for Thermal Power Engineering, Zhejiang University, Hangzhou 310027, China
}

Received December 15, 2011; accepted February 3, 2012

\begin{abstract}
We numerically simulate the hydrodynamic interaction of aerosol particles due to the acoustic wake effect under the Oseen flow condition. Attraction is found for two nearby particles with an orientation angle of 0 to $50^{\circ}$ with respect to the acoustic field, and weak repulsion is found outside this range. Good agreement is obtained between the numerical results and experiments in the literature. We study the influence of particle size, sound wave frequency and the particle separation. The result shows that the acoustic wake effect plays a significant role in acoustic agglomeration. It could be either the major agglomeration mechanism of monodisperse aerosols or the major refill mechanism for polydisperse aerosols to supplement orthokinetic interaction.
\end{abstract}

acoustic agglomeration, aerosol particle, hydrodynamic interactions, acoustic wake effect, Oseen region

Citation: Zhang G X, Liu J Z, Wang J, et al. Numerical simulation of acoustic wake effect in acoustic agglomeration under Oseen flow condition. Chin Sci Bull, 2012, 57: 2404-2412, doi: 10.1007/s11434-012-5212-1

Acoustic agglomeration is a process in which intense sound waves produce relative motions and collisions among aerosol particles. It can significantly shift the particle size distribution of an aerosol from smaller to larger sizes in a short time of the order of $1 \mathrm{~s}$. Acoustic agglomeration has potential use in air pollution control as an aerosol preconditioning process to enhance the performance of conventional particle filtering devices, which are inefficient for retaining particles smaller than $2.5 \mu \mathrm{m}$ [1].

Acoustic agglomeration is governed by complex interaction mechanisms. The most significant ones are orthokinetic and hydrodynamic interactions [2]. Orthokinetic interaction is the most obvious mechanism. Particles with different sizes are entrained differently into the oscillating motion of the medium because of the differences in particle inertia. The relative motion leads to the approach and collision between particles. In one acoustic cycle, each larger particle sweeps a certain volume by its motion relative to smaller particles, and collects all the smaller particles in it. This

*Corresponding author (email: zhangguangxue@gmail.com) volume is defined as the agglomeration volume. The mechanism of orthokinetic interaction was well summarized by Mednikov [3] and improved by other researchers [4-6]. Nevertheless, this mechanism cannot explain agglomeration of monodisperse aerosols or the way in which the agglomeration volume is refilled once it is emptied after one cycle.

Hydrodynamic mechanisms are those which produce particle interactions through the surrounding medium because of hydrodynamic forces and the asymmetry of the flow field around the particle [1]. These mechanisms are generally considered to be the main acoustic agglomeration mechanisms for monodisperse aerosols because they are also active for same-sized particles [7]. Hydrodynamic mechanisms mainly include mutual radiation pressure and the acoustic wake effect $[5,6,8,9]$. The mutual radiation pressure is the effect due to the nonlinear interactions produced between the particle scattering wave and the incident field. However, so far, there is still no experiment to confirm this effect as a cause of agglomeration, and theoretical studies show that the mutual radiation pressure is much less important than the acoustic wake effect in acoustic agglomeration [9]. 
The acoustic wake effect is based on the asymmetry of the flow field around a moving particle [8]. Considering two closely spaced particles oscillating in the acoustic field, the leading one will disturb the fluid and produce a wake behind itself. The wake, termed the acoustic wake, leads to a pressure reduction in the area behind the leading particle. If the second particle travels in this acoustic wake, it experiences a drag reduction and moves at an accelerated speed towards to the leading one [10]. The same effect occurs in the next acoustic half-cycle, but the roles of both particles change. As a consequence, the two particles converge within several acoustic cycles and eventually collide.

A theory to describe the acoustic wake effect based on Oseen flow fields was first proposed by Pshenai-Severin [11] for two same-sized particles aligned along the direction of the sound wave. Dianov et al. [12] extended the theory to include the interactions between differently sized particles and derived an analytical solution. However, both models are limited to particles aligned along the direction of the sound wave. Tiwary and Reethof [13] calculated the trajectories of particles due to the acoustic wake effect using numerical methods by neglecting the unsteady term. They concluded that the interaction velocities could be a major mechanism for refilling the agglomeration volume during acoustic agglomeration. The mechanism of the acoustic wake effect was experimentally verified by Hoffmann and Koopmann [8] using microscopic photography. Recently, a series of studies were performed by Gonzalez et al. [14] using analytical and numerical methods to calculate the interaction velocities of particles based on this effect, and the results were compared with experiments [9]. Although the acoustic wake effect has been found to be an important hydrodynamic mechanism, the studies on the effect are still rare. In addition, the computational results mentioned above do not agree with each other and sometimes even contradict one another. Thus, more studies on the acoustic wake effect are necessary.

In this paper, we present a numerical simulation of the acoustic wake effect based on Oseen flow fields. The interaction velocity due to this effect is calculated for any arbitrary orientation of the particle center-line with respect to the direction of the sound wave. The results are compared with the experiments of Gonzalez et al. [9] and Hoffmann [15]. The influences of the main parameters, i.e. frequency, vibration velocity amplitude of the medium, particle size and initial separation are also investigated.

\section{Theoretical model and numerical scheme}

In an acoustic field, the particles of an aerosol are partially entrained into the oscillating motion of the gas medium. The velocity of an isolated particle, $u_{\mathrm{p}}$, and the slip-flow velocity around it, $V$, can be described by [12]

$$
u_{\mathrm{p}}=q U_{0} \sin (\omega t-\varphi) \text {, }
$$

$$
V=l U_{0} \cos (\omega t-\varphi)
$$

where

$$
\begin{aligned}
& q=\frac{q_{s}+h l_{s}^{2}}{\sqrt{1+2 h l_{s}^{2} q_{s}+h^{2} l_{s}^{4}}}, \\
& l=\frac{l_{s}}{\sqrt{1+2 h l_{s}^{2} q_{s}+h^{2} l_{s}^{4}}},
\end{aligned}
$$

are, respectively, the entrainment and slip coefficients of the particle in the Oseen regime, $q_{s}=1 / \sqrt{1+(\omega \tau)^{2}}$ and $l_{s}=$ $\omega \tau / \sqrt{1+(\omega \tau)^{2}}$ are, respectively, the entrainment and slip coefficients of the particle in the Stokes regime, $\omega$ is the angular frequency, $U_{0}$ is the vibration velocity amplitude of the medium, $\varphi=\tan ^{-1}\left[l_{s} /\left(q_{s}+h l_{s}^{2}\right)\right]$ is the particle phase delay, $\tau=2 \rho_{\mathrm{p}} R^{2} / 9 \mu_{\mathrm{g}}$ is the particle relaxation time, $R$ is the particle radius, $\rho_{\mathrm{p}}$ is the density of the particle, $\mu_{\mathrm{g}}$ is the dynamic viscosity of the gas, and $h=9 \rho_{\mathrm{g}} U_{0} / 2 \pi \rho_{\mathrm{p}} \omega R$.

Two close-by particles would mutually perturb their motion by means of their periodically varying acoustic wakes, which leads to deviations from the original particle trajectories. As a consequence, the fluid velocity around a particle is not only determined by the vibration velocity of the incident sound, but also by the acoustic wakes of the nearby particles. The fluid velocity at the position of particle $i$ is expressed as a combination of the vibration velocity of the incident sound and a perturbation velocity $v_{i k}$ due to the acoustic wake effect of the close-by particle $k$ [12]:

$$
u_{f i}=U_{0} \sin \omega t+v_{i k} .
$$

Radial and angular components of the perturbation velocity generated by particle $k$ at the location of particle $i$ can be described as [16]

$$
\begin{gathered}
v_{i k r}=\frac{A_{k}}{r^{2}}-\frac{A_{k}}{r^{2}} \mathrm{e}^{-\frac{r}{2 v}\left(\left|V_{k}\right|-V_{k} \cos \theta\right)} \cdot\left[1+\frac{r}{2 v}\left(\left|V_{k}\right|+V_{k} \cos \theta\right)\right], \\
v_{i k \theta}=-\frac{A_{k} V_{k}}{2 r v} \sin \theta \cdot \mathrm{e}^{-\frac{r}{2 v}\left(\left|V_{k}\right|-V_{k} \cos \theta\right)},
\end{gathered}
$$

where $r$ is the separation of the two particles, $v$ is the kinematic viscosity of the gas medium, $V_{k}$ is the slip-flow velocity of particle $k$ described by eq. (2), $\theta$ is the angle between the direction of the sound wave and the particle center-line (see Figure 1), and $A_{k}$ is given by

$$
A_{k}=\frac{3 v R_{k} V_{k}}{2\left|V_{k}\right|}\left(1+\frac{3 R_{k}\left|V_{k}\right|}{8 v}\right) \text {. }
$$

The motion of the two particles in a sound field is described by the system of equations [13] 


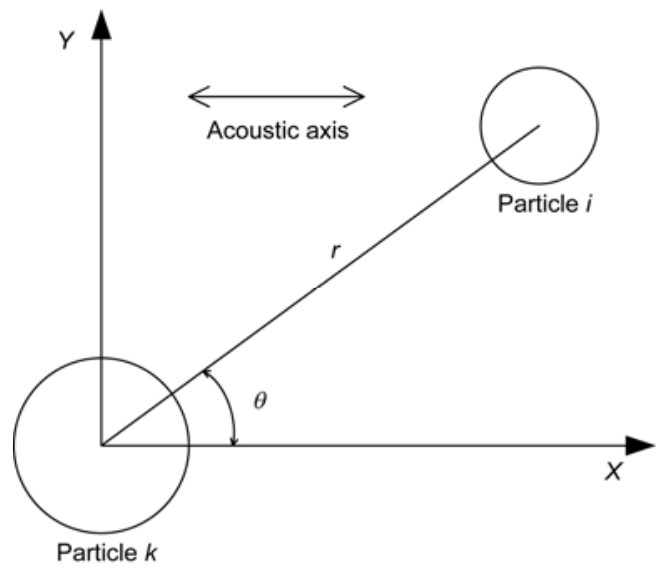

Figure 1 Two-particle coordinate system for the numerical simulation.

$$
\frac{\partial u}{\partial t}=\frac{C_{\mathrm{D}} R e V}{24 \tau}
$$

where $R e=2 R U_{0} l / v$ is the Reynolds number describing the flow condition of the particles, and $C_{\mathrm{D}}$ is the drag coefficient of the particles, given by

$$
C_{\mathrm{D}}= \begin{cases}\frac{24}{\operatorname{Re}}\left(1+\frac{3}{16} \operatorname{Re}\right), & (\operatorname{Re}<0.1), \\ \frac{24}{\operatorname{Re}}\left[1+0.1315 \operatorname{Re}^{\left(0.82-0.05 \log _{10} R e\right)}\right], & (0.1<\operatorname{Re}<10) .\end{cases}
$$

Because of the mutual perturbation velocity produced by the acoustic wake effect, particles will not return to the original position after one acoustic cycle. They may either attract or repel one another depending on their spatial orientation. The particle trajectories can be calculated by integrating the following equations:

$$
\begin{aligned}
& \frac{\partial x}{\partial t}=u_{x}, \\
& \frac{\partial y}{\partial t}=u_{y},
\end{aligned}
$$

where $u_{x}$ and $u_{y}$ are the $x$ - and $y$-components of the particle velocity, respectively. The expressions for converting the velocity defined in a spherical coordinate system to rectangular coordinates are

$$
\begin{aligned}
& u_{x}=u_{r} \cos \theta-u_{\theta} \sin \theta, \\
& u_{y}=u_{r} \sin \theta+u_{\theta} \cos \theta .
\end{aligned}
$$

The above equations constitute the theoretical model of the acoustic wake effect in acoustic agglomeration. We develop a numerical simulation method to solve these equations using 4th order Runge-Kutta integration. A time-step $h$ of $T / 1000$ is used to ensure high precision of the solutions.
Generally, the initial conditions of the interaction process, including the positions and velocities of the particles, should be given in a numerical simulation. However, these conditions are unknown here, so an alternative method is used. Both particles are assumed to be stationary at the beginning of the interaction process. Then they are entrained to the oscillation motion of the gas medium. Because the relaxation times of the particles are much smaller than the acoustic period, they achieve a steady state in a short time. However, during this time the particles deviate from the initial positions because of the transient effect. To overcome this, in the first few cycles we make both particles return to their initial positions at the end of every cycle. Therefore, after several cycles, particles will oscillate steadily around the initial positions. Then the particle velocities at the end of the last cycles are used as the initial velocities. In other words, we calculate the particle velocities and trajectories in the first few cycles just for obtaining the initial condition. It usually takes about four cycles to do that. After the initial conditions are obtained, the velocities and positions of particles as functions of time can be numerically solved.

\section{Results and discussion}

\subsection{Comparison with Dianov's analytical solution}

Dianov et al. [12] derived an analytical solution for particles aligned along the acoustic field by simplifying the perturbation velocity using Fourier expansion. The convergence velocity of two particles whose lines-of-center are parallel to the direction of the sound wave is given by

$$
u_{12}=\frac{3 U_{0}}{2 \pi r}\left(R_{1} l_{1}+R_{2} l_{2}\right) .
$$

Figure 2 shows the comparison between the results of our numerical simulation and the analytical treatment by Dianov et al. The calculations are carried out for differently sized pairs of particles in an acoustic field with $U_{0}$ of $1 \mathrm{~m} / \mathrm{s}$

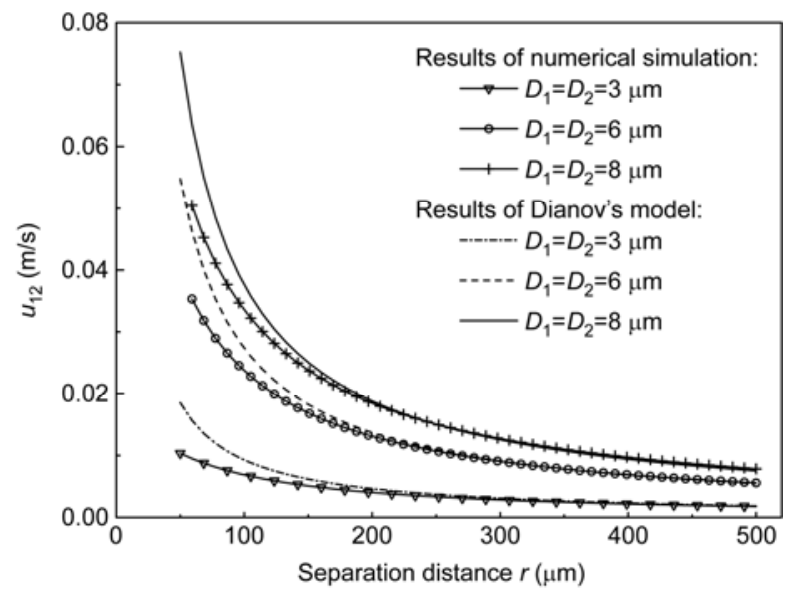

Figure 2 Comparison with Dianov's analytical solution. 
at a frequency of $2 \mathrm{kHz}$, and the particle density is $2400 \mathrm{~kg} / \mathrm{m}^{3}$. The comparison shows that the results agree very well for long separations, while the discrepancy increases rapidly with decreasing separation. This is because the term of $O\left(1 / r^{2}\right)$ discarded by Dianov is not negligible for small $r$. We will show that the results of the numerical simulation agree better with experimental data.

\subsection{Comparisons between the simulation and experi- ments}

To verify the numerical simulation, the computational results are compared with the experiments performed by Hoffmann and Koopmann [15] and Gonzalez et al. [9], in whose studies the particle trajectories in acoustic fields were visualized by means of CCD (charge-coupled device) video cameras. In these experiments, the micron-sized particles were dropped vertically under their gravitational forces in an acoustic field which vibrated horizontally, and photos of tuning fork agglomeration of particles were obtained.

Firstly, the results of the numerical simulation are compared with the experimental data of Hoffmann and Koopmann [15], as shown in Figure 3(a). They used soda lime glass sphere particles with a density of $2400 \mathrm{~kg} / \mathrm{m}^{3}$. The photo was captured in the acoustic field with a $U_{0}$ of $0.64 \mathrm{~m} / \mathrm{s}$ at a frequency of $800 \mathrm{~Hz}$. Initially the two particles aligned with the direction of the sound wave with a separation of $150 \mu \mathrm{m}$. It is shown that the two particles converged in the acoustic field and eventually collided. The solid line in Figure 3(a) is the particle trajectories calculated based on the average convergence velocity of the acoustic wake effect using equation (13). Figure 3(b) shows the particle trajectories calculated by our simulation and the particle cycle-averaged trajectories. It is clearly observed that Dianov's model overestimates the relative convergence velocity of the particles, and the results of the numerical simulation agree better with the experiment.

Figure 4 shows a similar comparison between our simulation results and the experimental data of Gonzalez et al. [9]. The photo was obtained in an acoustic field with a $U_{0}$ of
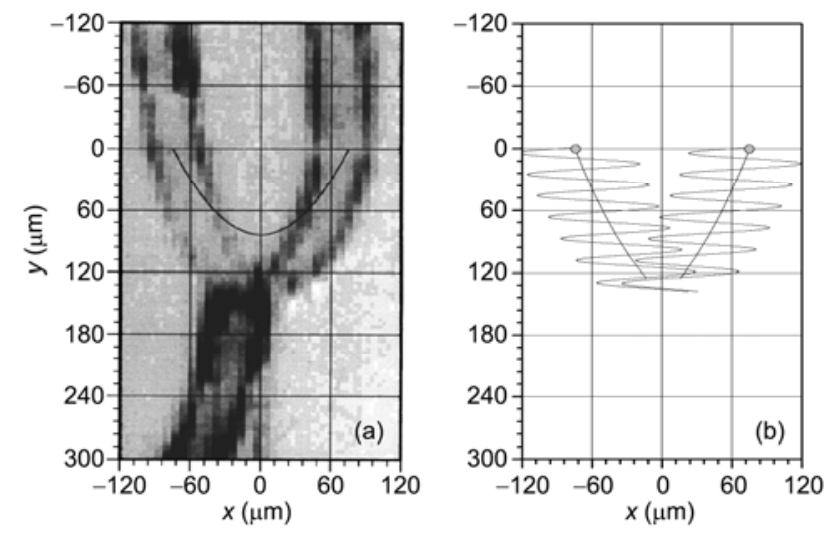

Figure 3 Comparison with experiment of Hoffmann and Koopmann [15].
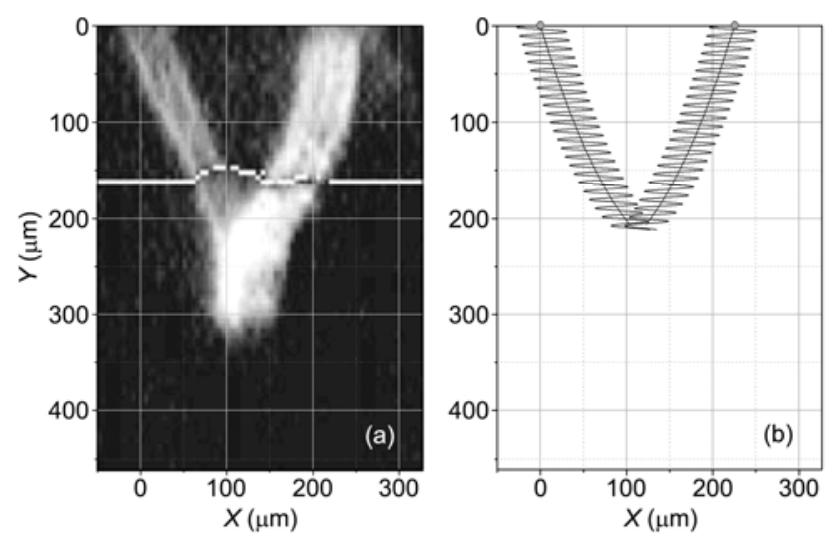

Figure 4 Comparison with experiment of Gonzalez et al. [9] for on-axis case.

$0.44 \mathrm{~m} / \mathrm{s}$ at a frequency of $900 \mathrm{~Hz}$. The two particles initially aligned along the direction of the sound wave with a separation of $207 \mu \mathrm{m}$. Figure 4(b) shows the particle trajectories calculated by our simulation, which agree very well with the experimental data.

The two comparisons above are for the case in which particles are aligned along the direction of the sound wave. Actually, agglomeration could also occur if the lines-ofcenter of two particles are not parallel to the direction of the sound wave. Two photos of the experiments carried out by Gonzalez et al. [9] are selected to verify the convergence velocity calculated by our simulation for particles whose center-line has angle $\theta_{0}$ with respect to the direction of the sound wave.

The agglomeration photo shown in Figure 5(a) was filmed under the conditions of $\theta_{0}=5^{\circ}$, frequency $f=700 \mathrm{~Hz}, U_{0}=$ $0.44 \mathrm{~m} / \mathrm{s}$ and initial separation $r_{0}=227.6 \mu \mathrm{m}$. Figure $5(\mathrm{~b})$ shows the computational results of the simulation under the same conditions. It is obvious from the figure that the numerical trajectories are similar to the experimental ones.

Figure 6(a) illustrates the agglomeration pattern of two particles with a larger angle of $\theta_{0}=15^{\circ}$, which was filmed under the conditions of $r_{0}=200 \mu \mathrm{m}, f=800 \mathrm{~Hz}$ and $U_{0}=$
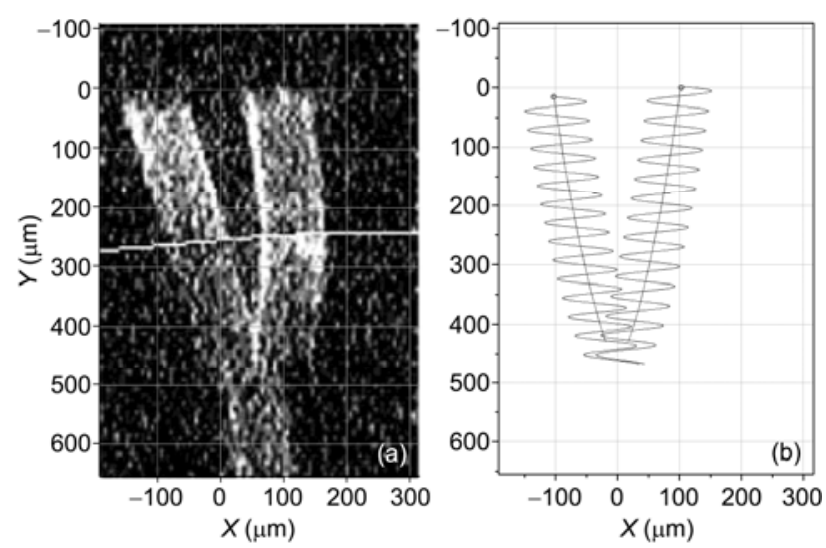

Figure 5 Comparison with experiment of Gonzalez et al. [9] for $\theta_{0}=5^{\circ}$. 

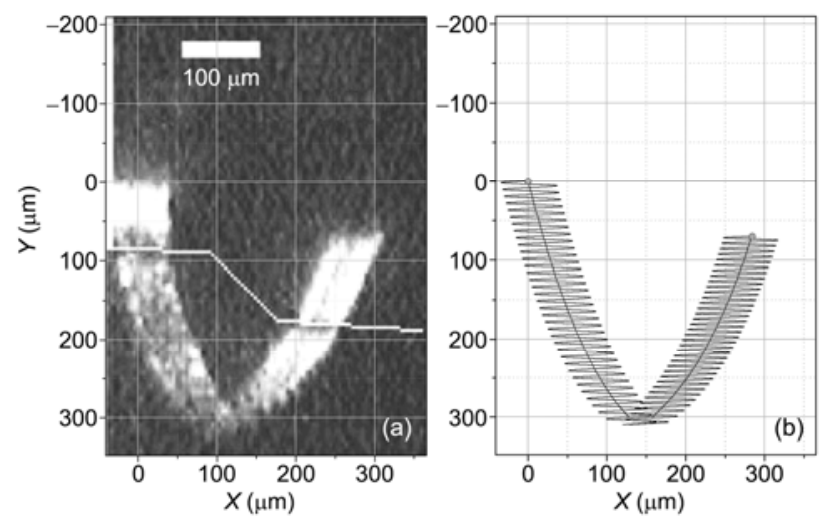

Figure 6 Comparison with experiment of Gonzalez et al. [9] for $\theta_{0}=15^{\circ}$.

$0.44 \mathrm{~m} / \mathrm{s}$. Again, numerical results shown in Figure 6(b) match well the experimental trajectories.

\subsection{Influence of the main parameters on interaction velocity}

(i) Particle orientation angle. The hydrodynamic interaction due to the acoustic wake effect between two particles varies greatly with different angles between the center-line of particles and the direction of the sound wave. Figure 7 shows the cycle-averaged interaction velocity between particles as a function of the angle $\theta$. We carried out the calculations for two same-sized particles with diameters of $6 \mu \mathrm{m}$ and a separation of $100 \mu \mathrm{m}$. The density of particles was $2400 \mathrm{~kg} / \mathrm{m}^{3}$ with $U_{0}=1 \mathrm{~m} / \mathrm{s}$ at a frequency of $2000 \mathrm{~Hz}$. In the figure, the $x$-axis refers to the direction of the sound wave.
A positive value of the convergence velocity indicates that particles approach each other, while a negative value means they move away from each other. We see that the interaction patterns are completely symmetrical, so we will focus on the angle ranging from 0 to $90^{\circ}$.

The maximum value of the $x$-component of the convergence velocity, $u_{12 x}$, is found for particles aligned along the direction of the sound wave. It decreases with increasing angle of orientation and drops to zero at about $\theta=45^{\circ}$. In the angular range of $45^{\circ}<\theta<90^{\circ}$, particles experience repulsion that reaches its maximum at approximately $\theta=60^{\circ}$. Compared to the strong attraction at small angles, the repulsion is much weaker. We find that for particles aligned perpendicularly to the direction of the sound wave $\left(\theta=90^{\circ}\right), u_{12 x}$ is zero, so the interaction between particles along the $x$-axis vanishes.

The $y$-component of the convergence velocity, $u_{12 y}$, has a maximum at about $\theta=30^{\circ}$. It becomes zero when particles are aligned along the direction of the sound wave. In the angular range of $60^{\circ}<\theta<90^{\circ}$, we find repulsion between particles that reaches its maximum at $\theta=90^{\circ}$.

Considering the acoustic wake effect as a probable cause of particle agglomeration in an acoustic field, the $r$-component of the convergence velocity, $u_{12 r}$, which could be calculated by eq. (12), is more useful. A positive value of $u_{12 r}$ represents the convergence of two particles. The value of $u_{12 r}$ as a function of the angle is plotted in Figure 7(c) and (f), which shows that $u_{12 r}$ has a maximum at $\theta=0^{\circ}$ and decreases as the angle increases. The attraction between particles drops to zero at about $\theta=50^{\circ}$, then turns to weak repulsion, which reaches its maximum at $\theta=90^{\circ}$. We observe that
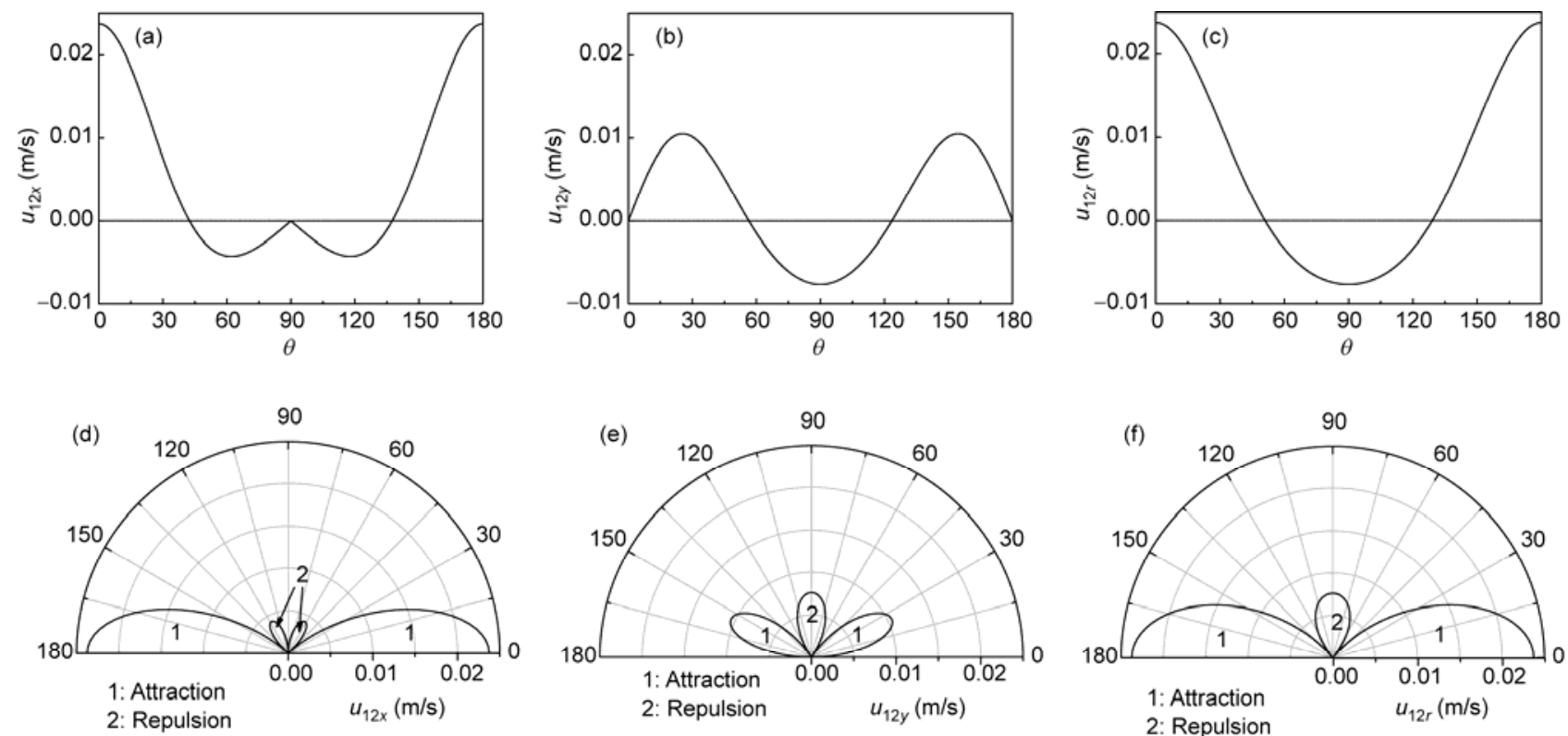

Figure 7 Interaction velocity as a function of angle. (a) $u_{12 x}$ as a function of $\theta$; (b) $u_{12 y}$ as a function of $\theta$; (c) $u_{12 r}$ as a function of $\theta$; (d) angular distribution of $u_{12 x}$; (e) angular distribution of $u_{12 y}$; (f) angular distribution of $u_{12 r}$. 
particles approach each other in the angular range of $0^{\circ}<\theta<50^{\circ}$ and repel each other in the angular range of $50^{\circ}<\theta<90^{\circ}$. The results of our numerical simulation coincide with the analytical solution of Dianov et al. [17] and the numerical results of Tiwary and Reethof [13], and contradict the analytical and numerical results of Gonzalez et al. [14], in whose studies particles aligned perpendicularly to the direction of the sound wave do not experience any interaction.

(ii) Particle separation. As shown in Figure 2, the convergence velocity of particles aligned along the direction of the sound wave increases rapidly with diminishing separation $r$. The relationship between them can be found in Dianov's model (eq. (13)), which shows that $u_{12}$ increases proportionately with $1 / r$. A similar relationship is obtained in our numerical simulation. For this reason, the particle agglomeration due to the acoustic wake effect represents a tuning fork pattern, as shown in Figures 3-6. In addition, we see from Figure 2 that smaller particles experience less attraction, which increases slowly with diminishing separation and becomes considerable only for short separations. However, the attraction between larger particles increases more rapidly with decreasing separation and could still be important in a long separation.

(iii) Frequency of sound wave. Figure 8 shows the convergence velocity as a function of sound frequency for different pairs of particles, separated by $150 \mu \mathrm{m}$ and subjected to an acoustic field with a velocity amplitude of $1 \mathrm{~m} / \mathrm{s}$. The computational results indicate that the convergence velocity increases with increasing sound frequency, but levels out to approach a constant value at high frequencies. As frequency increases, the slip-flow velocity increases because of the increasing slip coefficient. As a result, the Reynolds number increases and the asymmetry of the flow field around the particles is enhanced. The wakes of particles produced in the acoustic field are stronger, which leads to a higher convergence velocity. However, the slip coefficient approaches unity if the frequency is higher than a threshold value, then the particles are almost stationary and slip-flow velocity

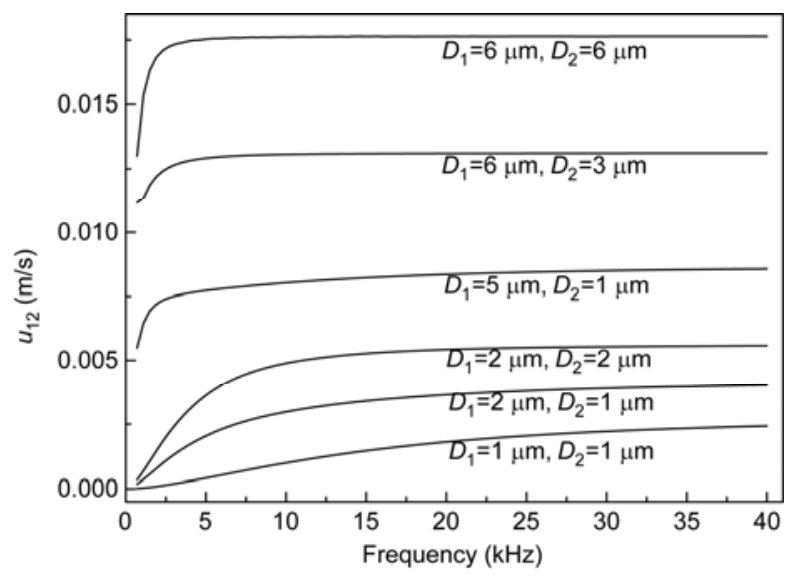

Figure 8 Dependence of the convergent velocity on the sound frequency. reaches a maximum constant value. A frequency above this threshold will not increase the convergence velocity any more. This threshold frequency can be determined by the slip coefficient (see eq. (4)). For larger particles, the threshold frequencies are lower. The calculations in this paper agree with the analytical and numerical results of Gonzalez et al. [14] and contradict the analytical solution of Dianov et al. [17], who found an optimum frequency at which the convergence velocity has a maximum for a pair of particles.

(iv) Particle size. Figure 9 shows the convergence velocity as a function of particle size. The calculations are carried out for particles aligned along the direction of the sound wave and initially separated by $200 \mu \mathrm{m}$ in an acoustic field with a velocity amplitude of $1 \mathrm{~m} / \mathrm{s}$. The diameter of one particle is kept constant, while the size of the other one varies. We find that the convergence velocity increases with increasing particle size. Similarly to the influence of the sound frequency, we attribute this increase to the higher slip coefficient for a larger particle. We see that the acoustic wake effect hardly acts as an effective interaction mechanism of agglomeration between small particles less than $2 \mu \mathrm{m}$.

(v) Vibration velocity amplitude of medium. The dependence of convergence velocity on the vibration velocity amplitude of the medium is shown in Figure 10. The computational results are obtained for particles aligned along the direction of the sound wave with an initial separation of 200 $\mu \mathrm{m}$. We observe that the convergence velocity increases a little faster than proportionally with increasing vibration velocity amplitude of the medium. That is because the wake caused behind the particle becomes stronger as velocity amplitude increases, and such dependence is nonlinear.

\subsection{Discussion of the computational results}

The hydrodynamic interaction due to the acoustic wake effect can be effective for particles separated by distances much larger than their acoustic displacements, and is not limited to the difference in particles sizes. Therefore, this

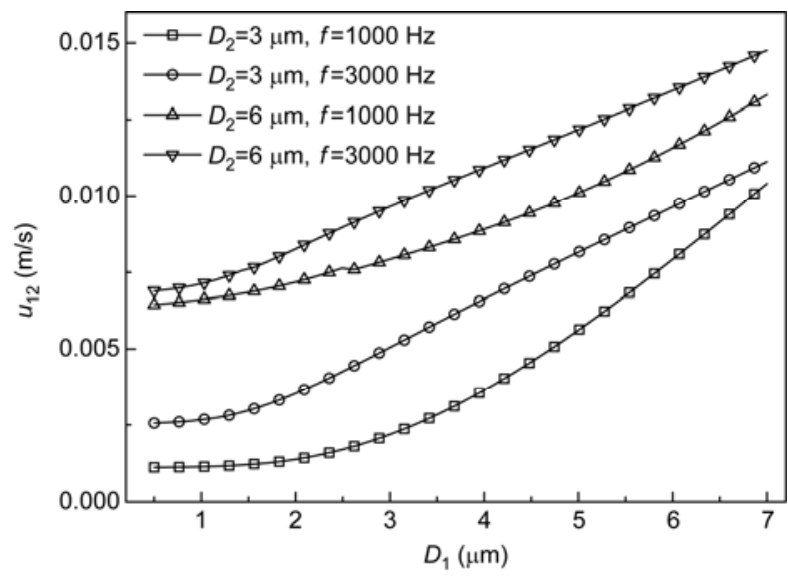

Figure 9 Dependence of the convergent velocity on the particle size. 


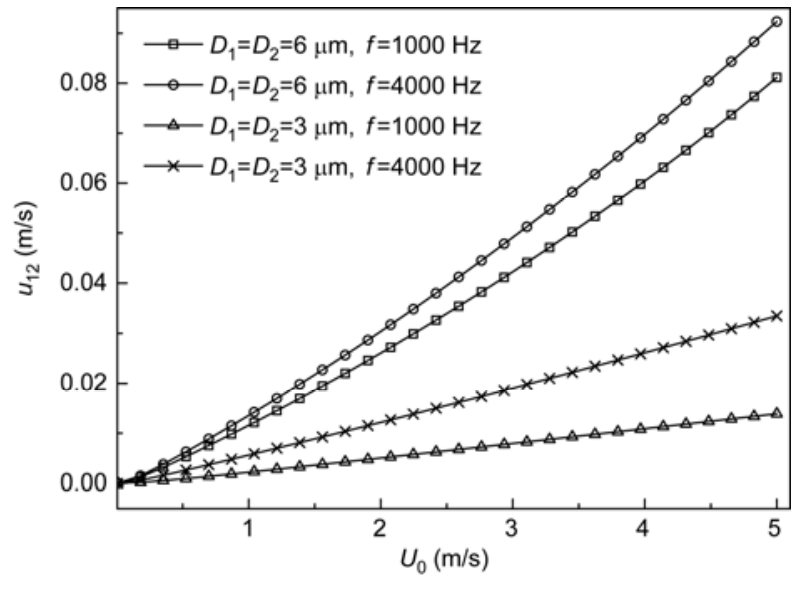

Figure 10 Dependence of the convergent velocity on $U_{0}$.

effect plays a significant role in acoustic agglomeration for both polydisperse and monodisperse aerosols.

(i) As the major agglomeration mechanism of monodisperse aerosols. The basis of orthokinetic interaction is the different entrainment coefficients of particles in the acoustic field. For monodisperse aerosols, orthokinetic interaction will not work because same-sized particles have the same entrainment coefficient and thus the relative motion among particles vanishes. However, experiments carried out by Shaw and Tu [18] show that monodisperse aerosols can also agglomerate rapidly in an acoustic filed. Moreover, the agglomeration patterns of monodisperse aerosols were observed directly using CCD video cameras [8,9]. Hence the only mechanisms to explain the phenomenon are the hydrodynamic interactions.

Figure 11 shows the agglomeration patterns of samesized particles aligned at six different initial angles of orientation. All these calculations are carried out for particles with diameters of $8 \mu \mathrm{m}$ and initial separations of $150 \mu \mathrm{m}$ in an acoustic field which vibrated horizontally at $U_{0}=0.5 \mathrm{~m} / \mathrm{s}$ and $2 \mathrm{kHz}$. In the figure, the $x$-axis refers to the direction of the acoustic field. The effect of gravity is neglected because the particles have the same diameters. Notice that the displacement amplitudes of the particles and the gas medium are $7.0 \mu \mathrm{m}$ and $39.8 \mu \mathrm{m}$, respectively, which are much shorter than their initial separation distances. When particles align along the direction of the sound wave $\left(\theta_{0}=0^{\circ}\right)$, they approach each other and finally collide after a short time of $10.6 \mathrm{~ms}$. At $\theta_{0}=30^{\circ}$, a similar attraction pattern occurs, but it takes $14 \mathrm{~ms}$ for the particles to collide. At $\theta_{0}=50^{\circ}$, as shown in Figure 7(c) and (f), the convergence velocity $V_{r}$ is almost zero. However, the $\theta$-component of the convergence velocity $V_{\theta}$ makes the particles rotate a little around the center of their center-line towards smaller $\theta$. Then they enter the attraction zone and finally collide after a time of $25 \mathrm{~ms}$. At $\theta_{0}=60^{\circ}$, the particles are in the repulsion zone
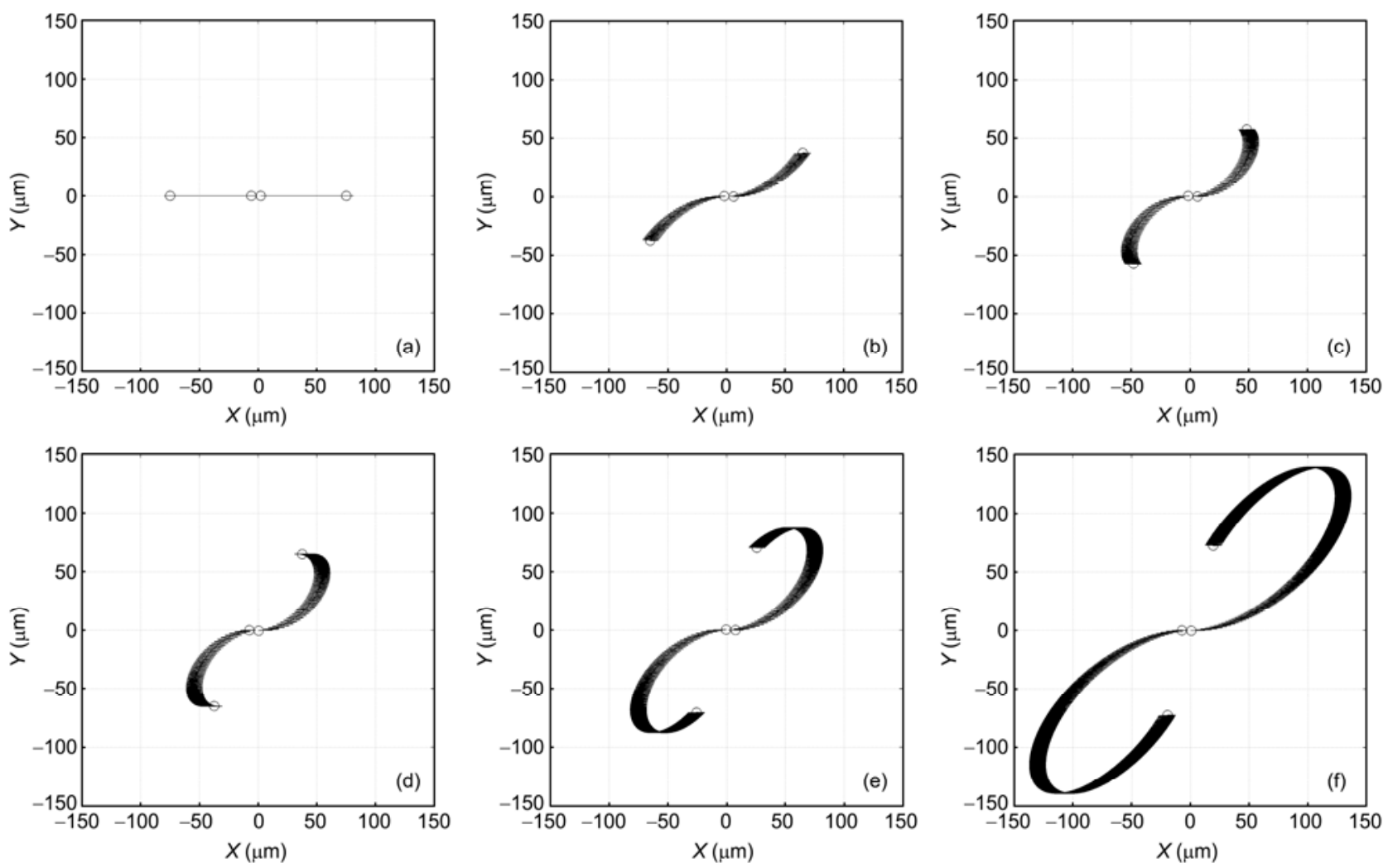

Figure 11 Agglomeration patterns of particles with varied initial angle of orientation. $D_{1}=D_{2}=8 \mu \mathrm{m}, R_{0}=150 \mu \mathrm{m}, U_{0}=0.5 \mathrm{~m} / \mathrm{s}$ and $f=2 \mathrm{kHz}$. (a) $\theta_{0}=0^{\circ}$; (b) $\theta_{0}=30^{\circ}$; (c) $\theta_{0}=50^{\circ}$; (d) $\theta_{0}=60^{\circ}$; (e) $\theta_{0}=70^{\circ}$; (f) $\theta_{0}=75^{\circ}$. 
and thus move away from each other at the beginning while moving towards the attraction zone. After a few cycles, they enter the attraction zone and finally collide after $42.2 \mathrm{~ms}$. A similar agglomeration happens at $\theta_{0}=70^{\circ}$ and $\theta_{0}=75^{\circ}$, but takes longer times of $114.8 \mathrm{~ms}$ and $443.7 \mathrm{~ms}$, respectively. At an angle larger than $75^{\circ}$, agglomeration will still occur, but it takes a much longer time, which means the attraction due to the acoustic wake effect is not important any more. Therefore, our numerical results show that particle agglomeration could occur at an angular range larger than the attraction zone. Under our simulation conditions, the particles aligned at an angle smaller than $75^{\circ}$ could collide in less than $0.5 \mathrm{~s}$, so the acoustic wake effect could be a major mechanism in acoustic agglomeration of monodisperse aerosols.

(ii) As the major refill mechanism. For polydisperse aerosols, orthokinetic interaction could be the dominant mechanism of agglomeration [19]. In the orthokinetic mechanism, every big particle in an aerosol, termed a core particle, will collect all other small particles within its agglomeration volume in one acoustic cycle. Thus, after one cycle the agglomeration volume of the core particle is emptied. No further agglomeration will occur until new particles are transported into this volume. The mechanisms for refilling the agglomeration volume are referred to as refill mechanisms [8]. Many kinds of mechanisms, such as Brownian motion of aerosols, turbulent gas motion, and gravitational effect of aerosols, have been proposed as the refill mechanisms. However, the refill factor based on these mechanisms gives agglomeration rates several orders of magnitude lower than the experimentally observed rates $[3,13,20]$. Since the acoustic wake effect was revealed to play a significant role with micron-sized particles, some researchers $[5,6,8,13,20]$ tried to use it as the major mechanism to explain the rapid refilling in acoustic agglomeration.

The cycle-averaged trajectories of small particles around a core particle are shown in Figure 12. All the trajectories are obtained in an acoustic field with $U_{0}=0.5 \mathrm{~m} / \mathrm{s}$ at $2500 \mathrm{~Hz}$ in a period of ten acoustic cycles. The diameters of the small particles and the core particle are $2 \mu \mathrm{m}$ and $8 \mu \mathrm{m}$, respectively. The hollow circle of each trajectory represents its initial position. Strong attraction is found for particles with an initial angle less than $50^{\circ}$. Small particles approach the core particle quickly in this zone. Small particles outside the attraction zone, i.e. the repulsion zone, move away from the core particle and are pushed to the attraction zone simultaneously. After a while, they reach the attraction zone and start to approach the core particle. We see that the attraction between the core particle and the majority of small particles near it is quite strong. Thus, the acoustic wake effect could be the major refill mechanism in acoustic agglomeration.

The refill mechanism proposed in this paper is different from the others which are also based on the acoustic wake effect, as shown in Figure 13. In the studies of Tiwary and Reethof $[13,20]$, strong repulsion was found for most small

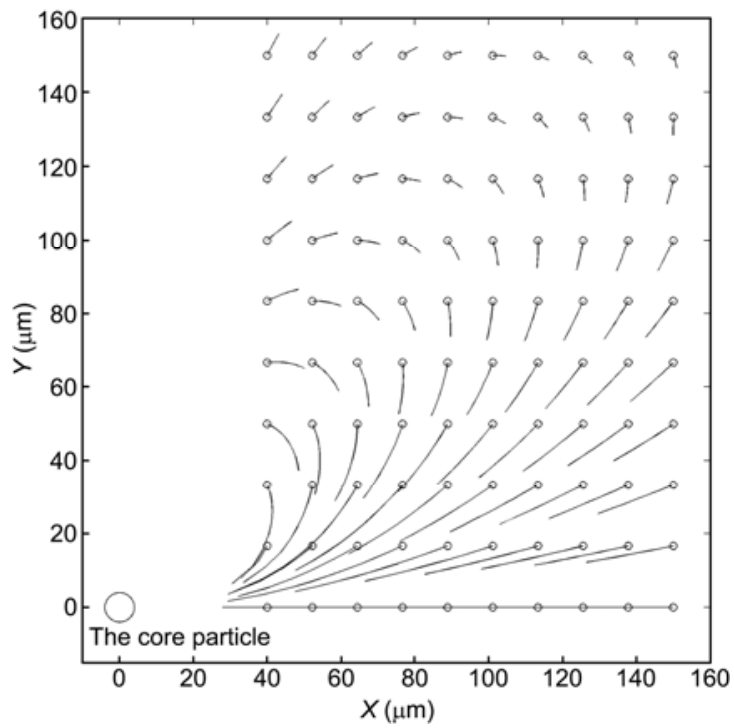

Figure 12 The cycle-averaged trajectories of small particles around a core particle in 10 cycles.

particles near a core particle. They concluded that small particles are pushed into the agglomeration volume by the adjacent large particles (see Figure 13(a)). Hoffmann [5] and Dong et al. [6] used Dianov's analytical solution as the major refill mechanism. As mentioned before, this solution is limited for particles aligned along the direction of the sound wave, so in their studies small particles enter the

(a)

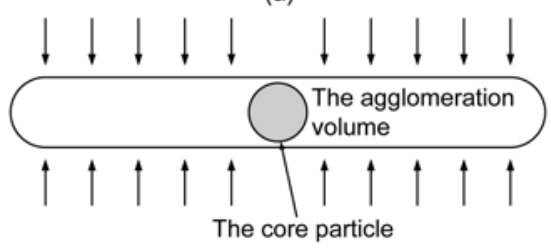

(b)
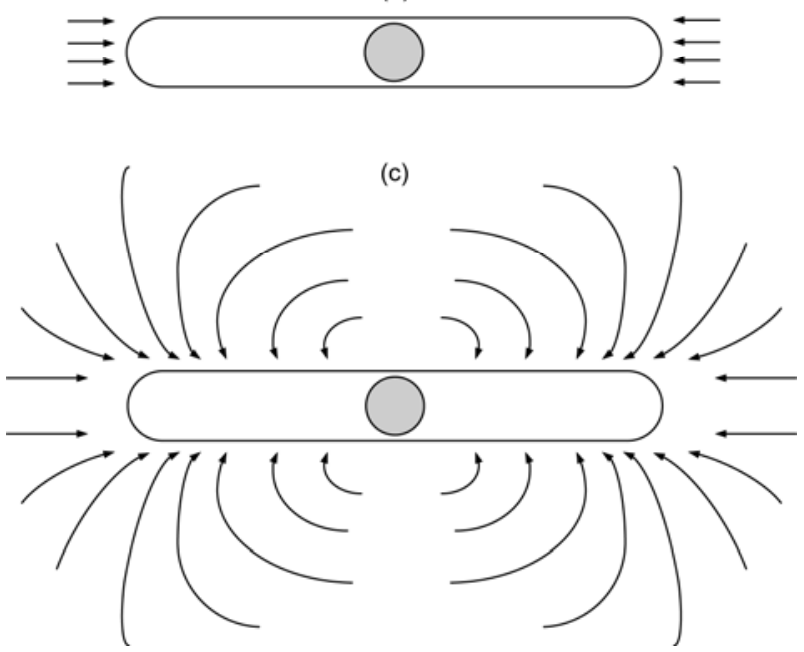

Figure 13 Schematics of the refill mechanisms proposed by Tiwary and Reethof [13,20] (a), Hoffmann [5] and Dong et al. [6] (b), and in this paper (c). 
agglomeration volume from the axial direction, as shown in Figure 13(b). In this paper, most small particles are pulled toward the core particle directly or gradually. Small particles could enter the agglomeration volume from various angles, as shown in Figure 13(c). Obviously, the refill mechanism proposed in this paper is more effective than the other two.

\section{Conclusion}

We carried out a numerical study to describe the acoustic wake effect in acoustic agglomeration. Good agreement was found between calculations and experiments by other researchers. The hydrodynamic interaction due to the acoustic wake effect varies with the orientation angle of particles respect to the direction of the acoustic field. Maximum attraction is found between particles aligned along the direction of the sound wave, while weak repulsion is found for particles aligned perpendicularly to that direction. The convergence velocity between two particles increases with increasing particle size and diminishing separation. It increases with increasing sound frequency and then approaches a constant value.

The result shows that the acoustic wake effect plays a significant role in acoustic agglomeration. It could be either the major agglomeration mechanism of monodisperse aerosols or the major refill mechanism for polydisperse aerosols to supplement the orthokinetic interaction.

This work was supported by the Zhejiang Natural Science Foundation (Y1100299). The authors thank Ms. Zhang Lili for helpful discussions and grammar check.

1 De Sarabia E R F, Gallego-Juarez J A, Rodriguez-Corral G, et al. Application of high-power ultrasound to enhance fluid/solid particle separation processes. Ultrasonics, 2000, 38: 642-646

2 Sheng C D, Shen X L. Modelling of acoustic agglomeration processes using the direct simulation monte carlo method. J Aerosol Sci, 2006, 37: 16-36

3 Mednikov E P. Acoustic Coagulation and Precipitation of Aerosols.
New York: Consultants Bureau, 1965

4 Cheng M T, Lee P S, Berner A, et al. Orthokinetic agglomeration in an intense acoustic field. J Coll Interf Sci, 1983, 91: 176-187

5 Hoffmann T L. An extended kernel for acoustic agglomeration simulation based on the acoustic wake effect. J Aerosol Sci, 1997, 28: 919-936

6 Dong S Z, Lipkens B, Cameron T M. The effects of orthokinetic collision, acoustic wake, and gravity on acoustic agglomeration of polydisperse aerosols. J Aerosol Sci, 2006, 37: 540-553

7 Riera E, Gallego-Juarez J A, Mason T J. Airborne ultrasound for the precipitation of smokes and powders and the destruction of foams. Ultrason Sonochem, 2006, 13: 107-116

8 Hoffmann T L, Koopmann G H. Visualization of acoustic particle interaction and agglomeration: Theory and experiments. J Acoust Soc, 1996, 99: 2130-2141

9 Gonzalez I, Gallego-Juarez J A, Riera E. The influence of entrainment on acoustically induced interactions between aerosol particlesAn experimental study. J Aerosol Sci, 2003, 34: 1611-1631

10 Hoffmann T L. Environmental implications of acoustic aerosol agglomeration. Ultrasonics, 2000, 38: 353-357

11 Pshenai-Severin S. On the convergence of aerosol particles in a sound field under the action of the oseen hydrodynamic forces. Dokl Akad Nauk SSSR, 1959, 125: 775-778

12 Dianov D B, Podol'skii A A, Turubarov V I. Calculation of the hydrodynamic interaction of aerosol particles in a sound field under Oseen flow conditions. Soviet Phys Acoust, 1968, 13: 314-319

13 Tiwary R, Reethof G. Hydrodynamic interaction of spherical aerosol particles in a high intensity acoustic field. J Sound Vibrat, 1986, 108: 33-49

14 Gonzalez I, Hoffmann T L, Gallego-Juarez J A. Theory and calculation of sound induced particle interactions of viscous origin. Acustica, 2000, 86: 784-797

15 Hoffmann T L, Koopmann G H. Visualization of acoustic particle interaction and agglomeration: Theory evaluation. J Acoust Soc, 1997, 101: 3421-3429

16 Nair R. Effects of high intensity acoustic fields on sulfur dioxide capture and particle agglomeration in pulverized coal combustors. Ph. D. Thesis. Pennsylvania: Pennsylvania State University, 1996

17 Dianov D B, Podol'skii A A, Turubarov V I. Analysis of the hydrodynamic aggregation of aerosol particles in a sound field under stokes flow conditions. Soviet Phys Acoust, 1967, 13: 37-40

18 Shaw D T, Tu K W. Acoustic particle agglomeration due to hydrodynamic interaction between monodisperse aerosols. J Aerosol Sci, 1979, 10: 317-328

19 Liu J Z, Zhang G X, Zhou J H, et al. Experimental study of acoustic agglomeration of coal-fired fly ash particles at low frequencies. Powder Tech, 2009, 193: 20-25

20 Tiwary R, Reethof G. Numerical simulation of acoustic agglomeration and experimental verification. J Vibrat Acoust Stress Rel Design, 1987, 109: 185-191

Open Access This article is distributed under the terms of the Creative Commons Attribution License which permits any use, distribution, and reproduction in any medium, provided the original author(s) and source are credited. 